\title{
Gravity Effect on Electrical Field Generation and Charge Carriers Transfer During Combustion Synthesis of Sulfides
}

\author{
A. A. Markov ${ }^{1, *}$, I. A. Filimonov ${ }^{2}$, K. S. Martirosyan ${ }^{3}$ \\ ${ }^{1}$ Institute for Problems in Mechanics, Russian Academy of Sciences, Moscow, 119526, Russia \\ ${ }^{2}$ Institute of Structural Macrokinetics and Materials Science (ISMAN), Russian Academy of Sciences, Chernogolovka, \\ Moscow, 142432, Russia \\ ${ }^{3}$ Department of Physics and Astronomy, University of Texas at Brownsville, Brownsville, 78520 USA \\ *Corresponding Author: a_a_markov@yahoo.com
}

Copyright (C) 2014 Horizon Research Publishing All rights reserved.

\begin{abstract}
The effect of gravity on the electric potential generated by the combustion synthesis of zinc sulfide is analyzed using the numerical simulation. Recent experimental studies on generation of electric voltage during combustion synthesis of zinc sulfide $(\mathrm{ZnS})$ have revealed high voltage signals $(4 \mathrm{~V})$ with duration about $1 \mathrm{~s}$, which are much higher than those produced by the gas-solid and solid-solid combustion reactions studied previously. These data have raised the question about mechanism of such a phenomenon. In our previous work we developed a novel (distributed) model describing the electric potential generation during combustion synthesis of sulfides (CSS) that didn't count the effect of gravity. In this paper the simulations of heat - mass transfer, charge carriers motion, and voltage profiles taking into account the Earth gravity effect. The simulations confirms that the gravitation force strongly affects the emission of negatively charged sulfur ions as well as electrons and has a significant impact on the amplitude and temporal evolution of the combustion induced voltage. The voltage reduction up to four times has been observed numerically in the case when gravity acts in the direction coincident to that of the propagating combustion wave. Vice versa, the significant acceleration of the combustion and the voltage amplification due to the advection is simulated when gravity acts in the direction opposite to that of the propagating combustion wave.
\end{abstract}

Keywords Combustion Synthesis, Zinc Sulfide Reaction, Electrical Field Generation, Charge Carriers

\section{Introduction}

Self-propagating High-temperature Synthesis (SHS) can be accompanied by various electrical phenomena. High temperature gas-solid reactions occurring during SHS produce, in some cases, a high concentration of electrical charge carriers, which may generate, in turn, various electrochemical and physical effects. Electrical fields generated by high-temperature chemical reactions have been observed in the gas phase surrounding burning titanium, zirconium, and iron single particles [1] for the following reactions $\mathrm{Ti}-\mathrm{O}_{2}, \mathrm{Zr}-\mathrm{O}_{2}, \mathrm{Fe}-\mathrm{O}_{2}, \mathrm{Ti}-\mathrm{N}_{2}$ and $\mathrm{Al}-\mathrm{Ni}$ inside compacted samples [2-6]. Similarly, a voltage difference of about $1 \mathrm{~V}$ was detected during the several SHS of ferrites by inserting two electrodes into the sample [7-9]. The mechanism yielding these electrical fields has not yet been reliably established and this prevents an a-priori estimate of the magnitude of this electrical field and its potential impact.

The experiments have showed [7] that the generated voltage (order $1 \mathrm{~V}$ ) is significantly higher than the thermoelectric effect at the electrodes, which is of the order of $\mathrm{mV}$. Moreover, the voltage attained its maximum value well ahead of the time at which the maximum temperature was attained. This fact rules out the possibility that thermal emission was the major contributor to the observed voltage in experiments. The estimates of the electric current due to the thermal emission by the Richardson-Dashman equation $J_{e}=(1-\bar{k}) A_{0} T^{2} \exp (-\phi / K T)$, where $\bar{k}$ is the electron reflection coefficient, $\varphi$ is the metal or metal oxide work function and $\mathrm{A}=4 \pi \mathrm{e}_{\mathrm{e}} \mathrm{k}^{2} / \mathrm{h}^{3}$ (e = electron charge, $\mathrm{m}_{\mathrm{e}}=$ electron mass, $\mathrm{k}=$ Boltzmann's constant, $\mathrm{h}=$ Plank's constant) predicted thermal emission currents of the order of $10^{-2} \mathrm{~A} / \mathrm{cm}^{2}$ for the oxidation of Ti at $2000 \mathrm{~K}$.

Different rates of diffusion of charge carriers across the growing oxide shell create an electric double layer. The production of positive and negative charge carriers such as electron holes, oxygen ions, vacancies, and electrons, either at the reaction front and/or the particle surface, is a necessary but not sufficient condition for the generation of voltage by the reaction. The formation of electric voltage with some certain polarity and amplitude depends on the difference between the diffusive velocities of charge carriers [4]. The 
goal of this study was to determine the impact of gravity on the electric field generated during $\mathrm{Zn}-\mathrm{S}$ reactions, on the formation of the electric signals, their polarity, amplitude, and shape. While experimental determination of the impact of reactants' properties, composition, environment, and sample size is important but straightforward, the experimental finding of the chemical reaction features that determine the amplitude and temporal evolution of the electric and magnetic signals is much more intricate. The thermally uniform 1D system was investigated first numerically [10-13]. The experimental and theoretical aspects of combustion synthesis of complex composites are studied in [14-19]. The cooperative multiphase processes that take place in the course of chemical vapor deposition (CVD) as well as in submicron particle synthesis via combustion from complex oxides are simulated numerically in [17-22]. The micro and macro two level numerical technique developed by Markov [20] is generalized in the present paper to investigate numerically the complex electro and gas dynamic features of the CSS process. The two level theoretical approaches for CVD have been proposed originally in [23].

\section{Formulation of the CSS Model Structure}

\subsection{Microscopic Problem}

On a microscopic scale comparable with the size of a growing particle, let us consider the set of simultaneous chemical reactions formulated as follows:

$$
\sum_{k} k \cdot S \rightarrow S_{k}^{2-}(\text { gas / solid })+2 h^{+}(\text {solid })+(-\Delta H),
$$

at

$$
\begin{gathered}
\forall k<n, n=\{2,4,6,8\}, k \subset N, \\
Z n \rightarrow 2 e(\text { gas })+Z n^{2+}(\text { solid }) \\
S^{2-}(\text { gas })+Z n^{2+}(\text { solid }) \rightarrow Z n S(\text { solid }) \\
h^{+}+e^{-} \leftrightarrow \text { nil }
\end{gathered}
$$

Where reaction (4) reflects thermal annihilation and generation of electron- hole pairs in the solid product (see [24]). Here "nil" indicates the product states in which there are no defects and its crystalline structure is perfect. Eqs. (1-3) reflect the fact established now experimentally that a negatively charged gas is emitted during CSS. The gas phase is supposed to contain mainly sulfur ions and electrons chemically released by the reaction. It is known [24] that in a vapor phase sulfur forms the following neutral and stable cyclic compositions: $S_{n}, n=\{2,4,6,8\}$, which are destroyed at temperatures higher than $180^{\circ} \mathrm{C}$ and transformed to linear chain fragments having uncompensated bonds and charged respectively. Thus, at high temperatures $\left(T>\cong 180^{\circ} C\right.$ ) the sulfur vapors produced intensively above the boiling point are partially ionized and the ionization degree is determined by the ratio of the linear chain fragment generation rates to the rate of their recombination into the neutral cyclic compositions.

In the present paper we made computation for simplified kinetics as follows:

$$
S \rightarrow S^{2-}(\text { gas })+2 h^{+}(\text {solid }) \quad(-\Delta H)
$$

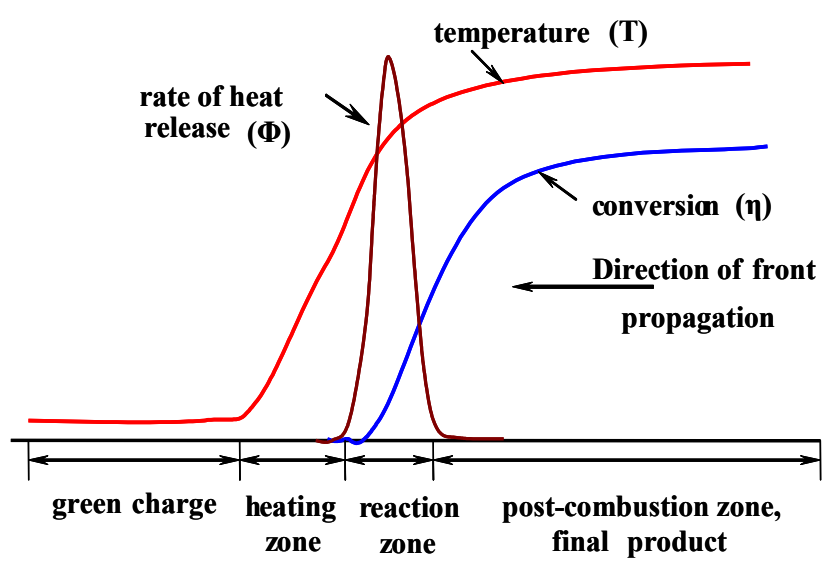

Figure 1. Schematic diagram of the Combustion Synthesis of Sulfides (CSS).

The schematic diagram of the sample and gaseous flow in CSS is shown in Figure 1. The thermal front propagation direction was defined from right to left.

\subsection{Macroscopic Problem}

While the CSS model equations (with the exception of kinetics on a microscopic level) are completely similar to those in CCSO model [17-18] and strongly different from those on a macroscopic level. The reason is that, in addition to mass transfer, they describe also transfer of charge carriers: ions, cations, electrons, and electron holes. The law of conservation of matter in the gas phase leads to the expression:

$$
\frac{\partial \chi \rho_{g}}{\partial t}+\nabla \cdot\left(\chi \rho_{g} u\right)=(1-\chi) J_{s \rightarrow g}^{\text {macro }}
$$

The equations for solid densities

$$
\frac{\partial \rho_{1 S}}{\partial t}=-J_{S}, \frac{\partial \rho_{2 S}}{\partial t}=\frac{M_{2 S}}{M_{1 S}} J_{S}
$$

The equation of impulses in the gas phase (the Navier-Stokes equation) is as follows:

$$
\begin{aligned}
& \frac{\partial \chi \rho_{g} u}{\partial t}+\nabla \cdot\left(\chi \rho_{g} u u\right)=S_{\mathbf{v}}-\frac{1}{\mathrm{Ma}^{2}} \nabla p+\frac{1}{\mathrm{Re}} \nabla \cdot \boldsymbol{\tau} \\
& -N_{E} \nabla\left(\varepsilon E^{2} / 2\right)+N_{E} \nabla \cdot \boldsymbol{\tau}_{E}+F_{B}
\end{aligned}
$$

The viscous tensor is as follows 


$$
\boldsymbol{\tau}=\mu\left\lceil\nabla \mathbf{u}+(\nabla \mathbf{u})^{\mathrm{T}}-\frac{2}{3}(\nabla \cdot \mathbf{u}) \mathbf{I}\right\rceil
$$

The electromagnetic effect is taken into account by the relation:

$$
\left(\tau_{\mathrm{E}}\right)_{\mathrm{ik}}=\varepsilon \mathrm{E}_{\mathrm{i}} \mathrm{E}_{\mathrm{k}}
$$

$\boldsymbol{\tau}_{E}$ is the part of the stress tensor taking into account the tensions provided by electric forces.

The full momentum equations include the gravity force and the porous resistance introduced by a (negative) momentum source term in the form:

$$
\left(\mathbf{S}_{\mathbf{V}}\right)_{i}=-u_{i} \kappa_{i}, \quad \kappa_{i}=\alpha_{i}|u|+\varsigma_{i}, i=1,2,3
$$

$F_{B}=G \rho_{g}(1-\chi)$ is the gravity force.

The electric field, electric current, and electric field stresses are as follows:

$$
\mathrm{E}=-\nabla \varphi, \mathrm{j}=-\sigma \nabla \varphi . \quad\left(\boldsymbol{\tau}_{E}\right)_{i k}=\varepsilon \nabla_{i} \varphi \nabla_{k} \varphi .
$$

Equation for the electric field potential (the Poisson equation) is written as:

$$
\Delta \phi+N_{\phi} q=0
$$

Charge density equation:

$$
\frac{\partial q}{\partial t}-N_{0} \nabla \cdot(\sigma \nabla \phi)=0
$$

Thermal conductivity equation taking into account the effect of chemical interaction and Joule dissipation has the form:

$$
\begin{aligned}
& \left(\chi \rho_{g} c_{g}+(1-\chi) \sum_{j} \rho_{j S} c_{j S}\right) \frac{\partial T}{\partial t}+\chi \rho_{g} c_{g} \mathbf{u} \cdot \nabla T= \\
& \nabla \cdot\left(\frac{\lambda}{P e_{T}} \nabla T\right)+Q^{\text {macro }}
\end{aligned}
$$

Where $\mathrm{Q}^{\text {macro }}=\mathrm{Q}^{\text {chem }}+\mathrm{Q}^{\mathrm{J}}$

Zero heat mass fluxes and zero velocities of gas flow in the reactor (lateral sample surface) as well as symmetry conditions at the reactor axis and initial conditions are imposed. Equations (6)-(12) are solved simultaneously with the microscopic Equation (5) At the ignition surface the prescribed initializing temperature is applied in numerical simulation.

The Frank-Kamenetsky dimensionless variables [25] (marked with tilde) are applied for the combustion front modeling

$$
\begin{gathered}
\tilde{x}_{i}=x_{i} / x_{*}, \tilde{t}=t / t_{*}, \tilde{u}_{i}=u_{i} / u_{*}, \\
\tilde{p}=p / p_{*}, i=1,2,3 \\
p_{*}=\frac{R \rho_{*} T_{*}}{M_{0}}, \rho_{*}=\rho_{S 0} \\
\tilde{\rho}_{g}=\rho_{g} / \rho_{*} \quad \tilde{\rho}_{j S}=\rho_{j S} / \rho_{*}, \\
\tilde{c}_{j S}=c_{j S} / c_{*}, \tilde{c}_{g}=c_{g} / c_{*}
\end{gathered}
$$

$$
\tilde{p}=\tilde{\rho}_{g}(1+\beta \tilde{T}), \frac{T}{T_{*}}=1+\beta \tilde{T}
$$

The main similarity parameters in combustion modeling are as follows $\beta=\frac{R T_{*}}{E}, \quad \gamma=\frac{c_{P} T_{*} \beta}{Q}$. Let us note that the Zeldovich Number $Z=\frac{E\left(T_{*}-T_{0}\right)}{R T_{*}^{2}}$ is equal to $Z=\frac{1}{\gamma}=\frac{Q}{c_{P} T_{*} \beta}$. The initial temperature is given as

$$
\tilde{T}_{0}=-\frac{1}{\gamma}
$$

\subsection{Estimates of the Gravity Effect}

First, we estimate the Froude number and thermal conductivity

We arrive at Froude number $G=\frac{g t_{*}{ }^{2}}{x_{*}}$ and temperature conductivity coefficient $\Psi=\frac{\lambda_{*}}{c_{*} \rho_{*}}$

Using Frank-Kamenetsky dimensionless variables, we obtain $\Psi=\frac{\mathrm{x}_{*}^{2}}{\mathrm{t}_{*}}$. For sulfur at $\mathrm{T}=368 \mathrm{~K}$, we find $x_{*} u_{*}=0.1$, thus $\frac{x_{*}^{2}}{t_{*}}=0.1, x_{*}=\frac{0.1}{u_{*}}, t_{*}=\frac{0.1}{u_{*}^{2}}$ The acceleration A of combustion wave and Froude Number $G$ are as follows

$$
A=\frac{x_{*}}{t_{*}^{2}}=10 u_{*}^{3} \quad G=\frac{9.8}{10 u_{*}^{3}} \text {. }
$$

Our estimates [19] show that $u_{*}=0.3 \div 30 \frac{\mathrm{m}}{\mathrm{c}}$. Therefore $G \approx 100 \div 1$ for slow and fast moving combustion fronts, respectively. The estimates [19] allow us to use the electro hydrodynamic (EHD) approximation in searching solution to the numerical problem. During calculation of the Maxwell equations, we also neglected displacement currents. This means that we consider electrical currents of the limited frequency only.

\section{Results and Discussion}

The low initial electric charge uniformly distributed in reactor was prescribed. The results of calculation for plane geometry are presented in Figures $1-8$. The main parameters are as follows

$$
\begin{gathered}
\beta=0.07, \quad \beta_{R}=100, \quad \alpha_{R}=0, \gamma=0.2, \quad \chi=0.5 \\
\operatorname{Re}=10, P e_{T}=10, \quad G=100, \mathrm{Ma}=0.1
\end{gathered}
$$




$$
\alpha_{i}=\alpha_{R}, \quad \beta_{i}=\beta_{R}, \quad i=1,2,3
$$

We consider the results of computation when gravity is the co directional to the thermal wave propagation (from the left side to the right side) as well as the case of in the anti directional gravity. The results of computations are compared to those ones neglecting the gravity effect and finally between themselves. The results of computation presented in Figures 2-8 refer to the given ignition temperature at the left side of the sample.

Figure 2, (a) shows the gravity effect when gravity force is co directional to the thermal wave propagation (from the left side to the right side) $G=100, \alpha_{R}=0, \beta_{R}=100$. The significant acceleration of the combustion front is found under gravitation Figures 2,(a) and is compared to the computation neglecting the gravity Figures 2,(b).
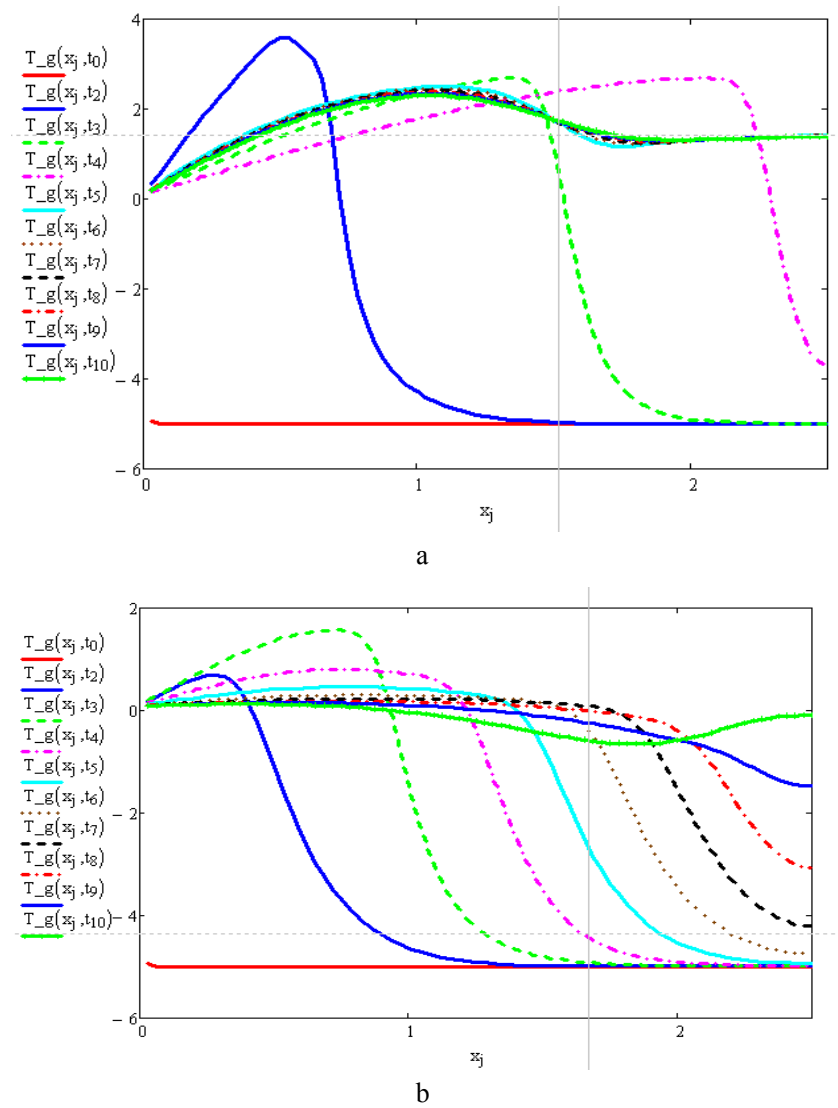

Figure 2. (a) - The gravity effect on temperature distribution along the sample at subsequent moments. Gravity is co directional to the thermal wave propagation (from the left side to the right side) $\mathrm{G}=100, \alpha_{R}=0, \beta_{R}=100$; (b) - The temperature distribution along the sample at subsequent moments with the neglected gravity.

The calculation of distribution of the velocity of sulfur ions, the vapors pressure, temperature and vapors density along the sample at $t_{2}$ and $t_{4}$ moments where $t_{4}>t_{2}$ are presented in Figure 3,(a-d). These data were obtained at the conditions where gravity force is co directional to propagating thermal wave at $G=100, \alpha_{R}=0, \beta_{R}=100$. It can be seen that the velocity of ions decreasing with the time Figure 3,(a), and the distribution of temperature along the sample shows wave propagation behavior Figure 3,(c). The calculation of distribution of the velocity of sulfur ions, the vapors pressure, temperature and vapors density along the sample at $t_{2}$ and $t_{4}$ moments where $t_{4}>t_{2}$ neglecting the gravity are presented in Figure 4,(a-d). It can be seen that computation neglecting the gravity force slightly changed the graphs.
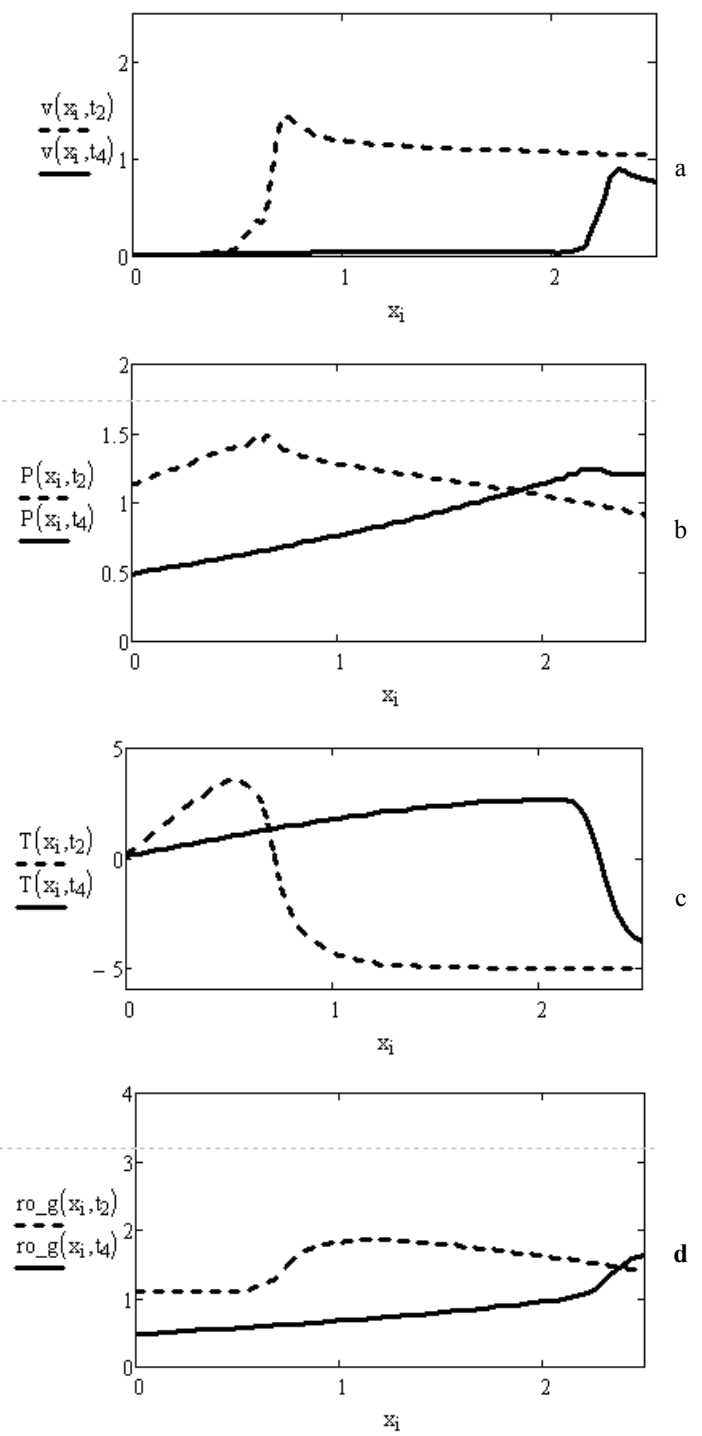

Figure 3. Gravity force is co directional to propagating thermal wave at $\mathrm{G}=100, \alpha_{\mathrm{R}}=0, \beta_{\mathrm{R}}=100$. (a) - Distribution of the velocity of sulfur ions along the sample at moments $\left(\mathrm{t}_{2}, \mathrm{t}_{4}\right) ; \mathrm{t}_{4}>\mathrm{t}_{2} ;(\mathrm{b})$ - The vapors pressure along the sample at the moments $\left(\mathrm{t}_{2}, \mathrm{t}_{4}\right) \mathrm{t}_{2}=12$, (dashed line), $\mathrm{t}_{4}=24$ (solid line); (c) Distribution of the temperature along the sample at moments $\left(t_{2}, t_{4}\right) ; t_{4}>t_{2} ;(d)$ - the vapors density along the sample at the moments $\left(\mathrm{t}_{2}, \mathrm{t}_{4}\right) \mathrm{t}_{2}=12$, (dashed line), $\mathrm{t}_{4}=24$ (solid line).

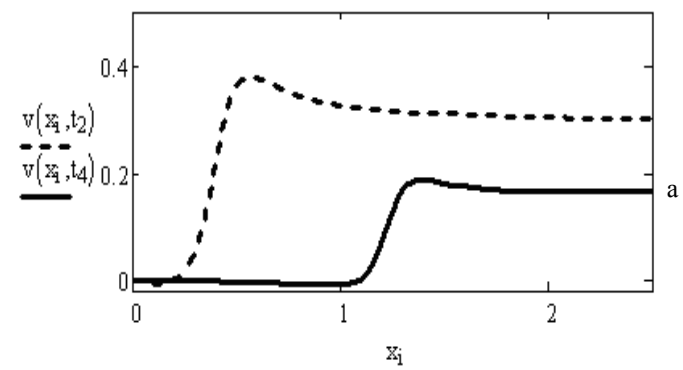



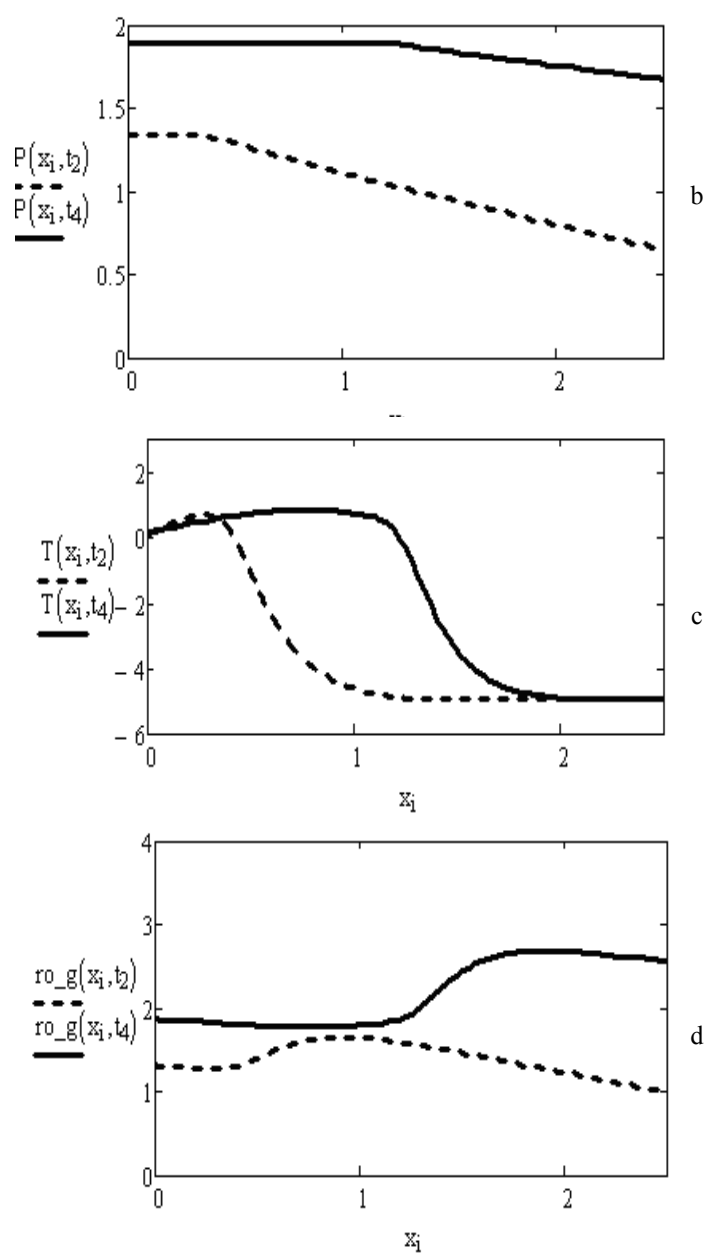

Figure 4. Computation neglecting the gravity: (a) - Distribution of the sulfur ions velocity along the sample at moments $\left.\mathrm{t}_{2}, \mathrm{t}_{4}\right) ; \mathrm{t}_{4}>\mathrm{t}_{2} ;(\mathrm{b})$ - The vapors pressure along the sample at the moments $\left(t_{2}, t_{4}\right) t_{2}=12$ (dashed line), $\mathrm{t}_{4}=24$ (solid line); (d) - Distribution of the temperature along the sample at moments $\left.\mathrm{t}_{2}, \mathrm{t}_{4}\right) ; \mathrm{t}_{4}>\mathrm{t}_{2} ;$ (c) - The vapors density along the sample at the moments $\left(\mathrm{t}_{2}, \mathrm{t}_{4}\right) \mathrm{t}_{2}=12$ (dashed line), $\mathrm{t}_{4}=24$ (solid line)

Figure 5 shows the distribution of the sulfur ions velocity, vapor pressure, temperature, and density along the sample axis at time moments $t_{2}=12$ (dashed) and $t_{4}=24$ (solid). In that case the gravity force acts oppositely to the thermal wave propagation $\mathrm{G}=-100, \alpha_{\mathrm{R}}=0, \beta_{\mathrm{R}}=100$.

Figure 6 illustrates distribution of the electric field intensity $\mathrm{E}$ and current density $\mathrm{J}$ along the sample axis at time moments $\mathrm{t}_{2}=12$ (dashed) and $\mathrm{t}_{4}=24$ (solid). Gravity acts along with the thermal wave propagation (from the left side to the right side) $\mathrm{G}=100, \alpha_{\mathrm{R}}=0, \beta_{\mathrm{R}}=100$.

Figure 7 illustrates distribution the electric field intensity $\mathrm{E}$ and current density $\mathrm{J}$ along the sample axis at time moments $\mathrm{t}_{2}=12$, (dashed) and $\mathrm{t}_{4}=24$, (solid). Computation neglecting the gravity force is done.

Figure 8 illustrates distribution the electric field intensity along the sample axis at subsequent time moments for the computation neglecting the gravity. Figure 8,(b) illustrates distribution the electric field intensity along the sample axis at subsequent time moments. Gravity force acts oppositely to the thermal wave propagation $\mathrm{G}=-100, \alpha_{R}=0, \beta_{R}=100$. When the gravity acts oppositely to the thermal wave propagation, the electric field strength is increasing up two times compared to the computation neglecting the gravity. Let us note the decreasing of electric field up to four times in the cases when gravity acts in the direction coincident to propagating combustion wave and vice versa for the gravity directed oppositely to the front propagation. The data showed in Figure 6-8 demonstrated that sulfur ions and electrons emanating chemically and the rate of solid transfer of the components is strongly limited by their diffusion in solids and is rather slow and the motion of sulfur vapors, which contain charged ions and electrons proceeds much faster, thus forming a vapor cloud charged negatively ahead of the combustion front. In that case the gravity significantly accelerates the wave propagation due to the advection transfer that becomes driving force for charge carriers. The numerical results obtained in Figures 6-8 are on the boundary between the combustion theory and electrochemistry. They allow estimating numerically the gravity contribution into the generated voltage and concluding which experimental scheme is the most preferable for reliable observation of the considered effect for possible application. These results may also have a contribution for combustion in microgravity as well as to the theory of electric sources. Nevertheless, the results are valid as an electro hydrodynamic (EHD) approximation and are obtained for the generated or applied voltage of the limited frequency (below150 GHz) [19].
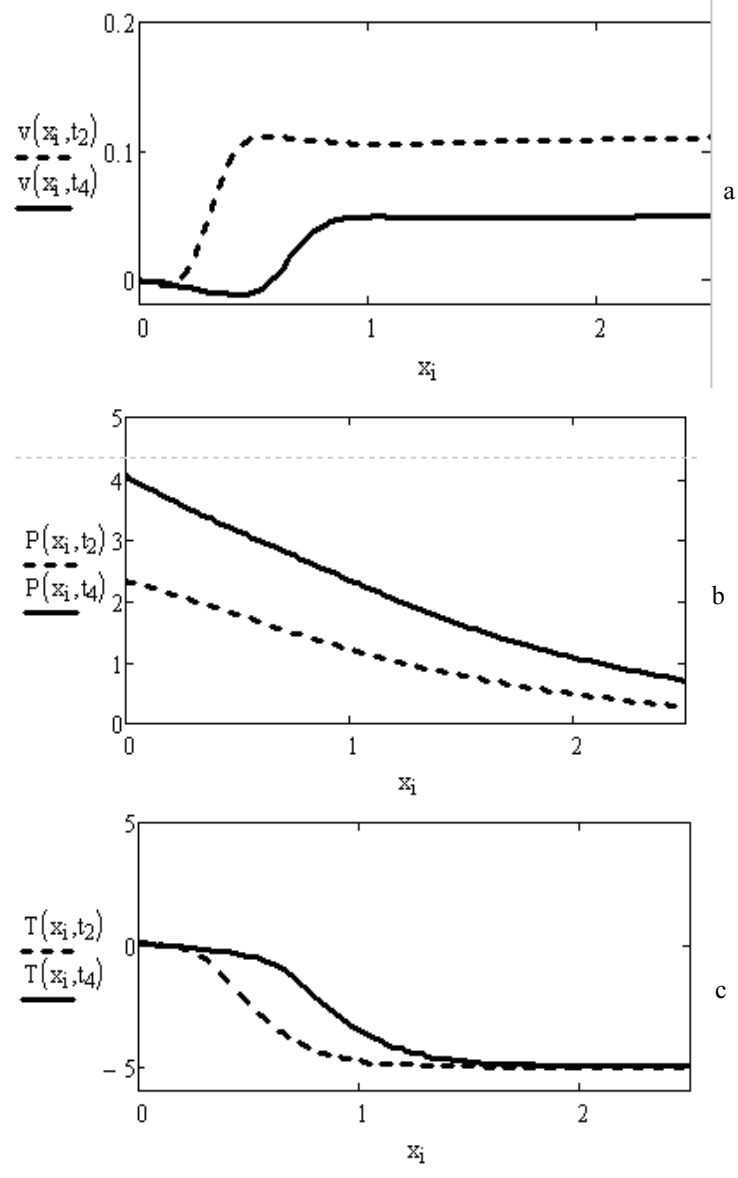


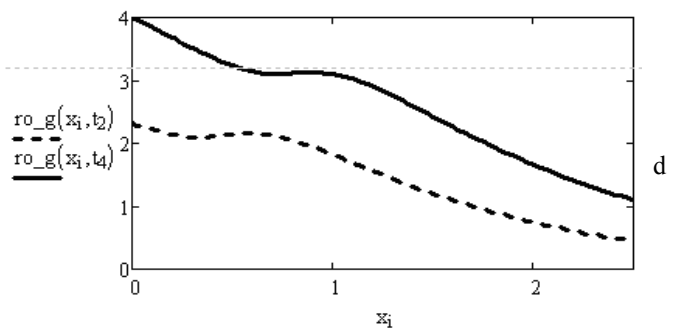

Figure 5. Gravity acts oppositely to thermal wave propagation. $\mathrm{G}=-100$, $\alpha_{R}=0, \beta_{R}=100$; (a) - Distribution of the sulfur ions velocity along the sample at moments $\left.\mathrm{t}_{2}, \mathrm{t}_{4}\right) ; \mathrm{t}_{4}>\mathrm{t}_{2} ;(\mathrm{b})$ - The vapors pressure along the sample at the moments $\left(t_{2}, t_{4}\right) t_{2}=12$ (dashed line), $t_{4}=24$ (solid line); (c) - Distribution of the temperature along the sample at moments $\left.\mathrm{t}_{2}, \mathrm{t}_{4}\right) ; \mathrm{t}_{4}>\mathrm{t}_{2} ;(\mathrm{d})$ - The vapors density along the sample at the moments $\left(\mathrm{t}_{2}, \mathrm{t}_{4}\right) \mathrm{t}_{2}=12$ (dashed line), $\mathrm{t}_{4}=24$ (solid line).
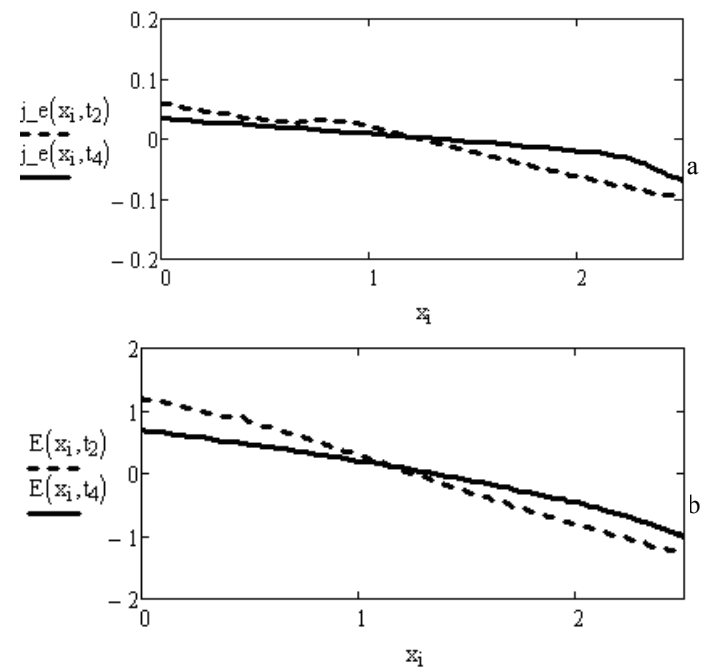

Figure 6. Gravity force acts coincidently to the thermal wave propagation (from the left side to the right side) $\mathrm{G}=100, \alpha_{\mathrm{R}}=0, \beta_{\mathrm{R}}=100$. (a) - Distribution of the electric field Intensity (E) along the sample at the moments $\mathrm{t}_{2}=12$, (dashed line) and $\mathrm{t}_{4}=24$ (solid line). (b) - The current density distribution along the sample at the moments $\mathrm{t}_{2}=12$, (dashed line) and $\mathrm{t}_{4}=24$ (solid line).
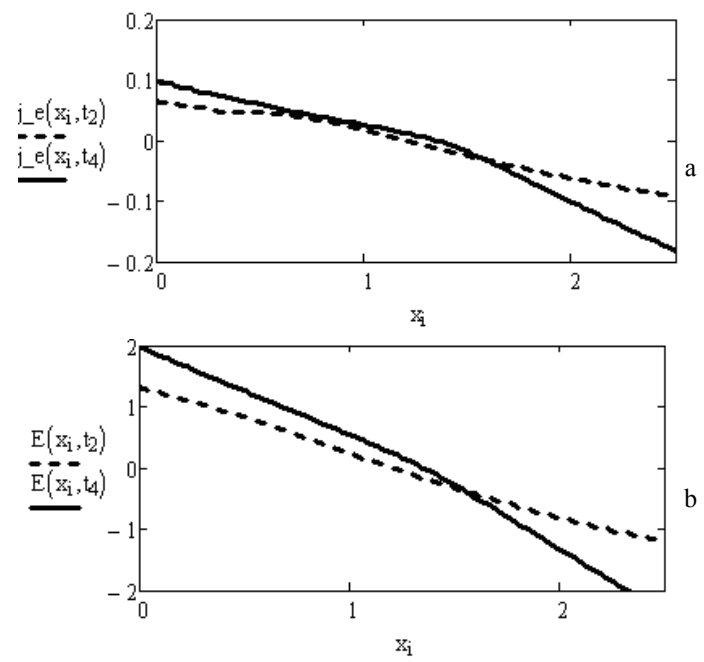

Figure 7. Computation neglecting the Gravity: (a) - Distribution of the electric field Intensity $(\mathrm{E})$ along the sample at the moments $\mathrm{t}_{2}=12$, (dashed line) and $\mathrm{t}_{4}=24$ (solid line); (b) - The current density distribution along the sample at the moments $\mathrm{t}_{2}=12$, (dashed line) and $\mathrm{t}_{4}=24$ (solid line)
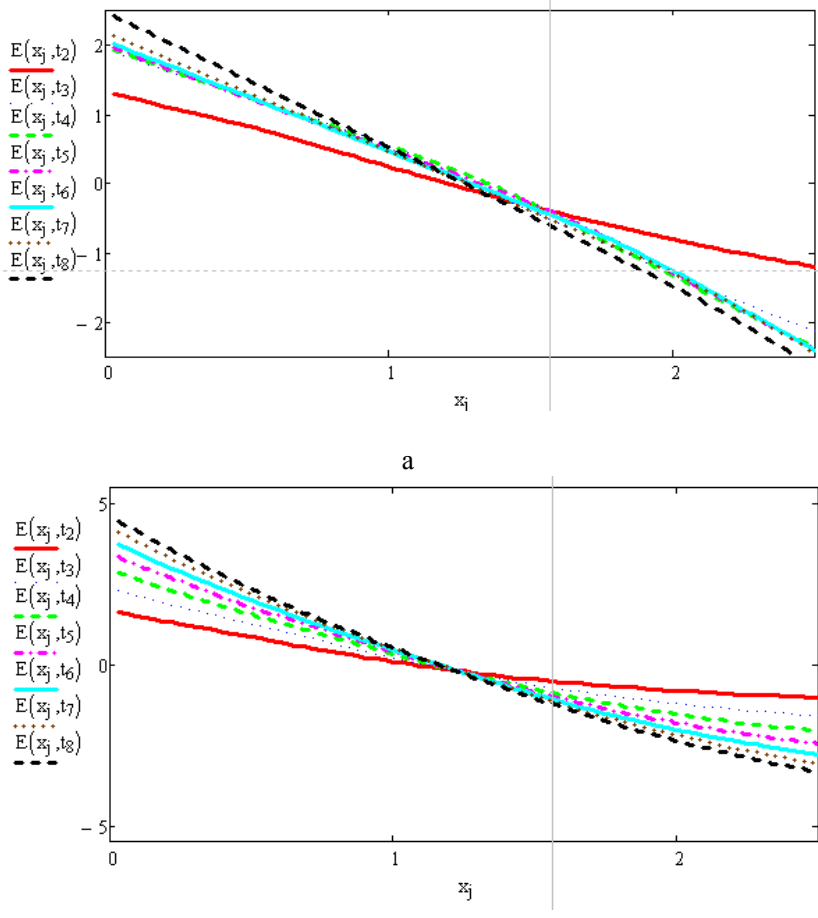

$\mathrm{b}$

Figure 8. (a) - Computation neglecting the gravity: the distribution the electric field intensity along the sample axis at subsequent time moments; (b) - Gravity acts oppositely to the thermal wave propagation $\mathrm{G}=-100, \alpha_{\mathrm{R}}=0$, $\beta_{\mathrm{R}}=100$.

\section{Conclusions}

The numerical study performed in this report lead to the conclusion that sulfur ions and electrons emanating chemically by the CSS and the self propagating high temperature front plays a key role in charge transfer through the reacting sample. While the rate of solid transfer of the components is strongly limited by their diffusion in solids and is rather slow and the motion of sulfur vapors, which contain charged ions and electrons proceeds much faster, thus forming a vapor cloud charged negatively ahead of the combustion front. The gravity significantly accelerates the combustion process due to the advection transfer that becomes more important compared to the diffusion. The electric field intensity is lower when the thermal wave propagates in the direction of gravity and vice versa when gravity acts oppositely to the thermal wave propagation. The result can be explained by the strong advection transfer of charged species for the acceleration caused by the gravity. Hot sulfur ions and electrons form a negatively charged gaseous cloud which flows up by advection and warms up the green charge zone of the CSS wave accelerating combustion and amplifying the electrical field.

\section{Acknowledgements}

We would like to acknowledge the financial support of 
this research by the Russian Foundation for Basic Research (grant no. 110800676) and the RAS Presidium Program N 26 (coordinated by academicians A.G. Merzhanov and V.A. Levin.

\section{Nomenclature}

$t_{*}=\frac{\exp \left(E / R T_{*}\right)}{k}$ reference time scale;

$x_{*}=\sqrt{\lambda t_{*} /\left(c_{*} \rho_{*}\right)}$ reference length scale;

$T^{*}=T_{\text {boil }}^{S}$ reference temperature;

$\beta=\frac{R T_{*}}{E}, \quad \gamma=\frac{c_{P} T_{*} \beta}{Q}$ main similarity parameters;

$\tilde{T}_{0}=-\frac{1}{\gamma}$ dimensionless initial temperature;

$$
\begin{gathered}
\tilde{x}_{i}=x_{i} / x_{*}, \tilde{t}=t / t_{*}, \tilde{u}_{i}=u_{i} / u_{*}, \\
\tilde{p}=p / p_{*}, i=1,2,3 \\
\tilde{\rho}_{g}=\rho_{g} / \rho_{*} \quad \tilde{\rho}_{j S}=\rho_{j S} / \rho_{*}, \\
\tilde{c}_{j S}=c_{j S} / c_{*}, \tilde{c}_{g}=c_{g} / c_{*} \\
\frac{T}{T_{*}}=1+\beta \tilde{T}
\end{gathered}
$$

dimensionless variables marked by tildes;

$C_{j} \quad$ mass fraction of species $j$, dimensionless;

$C_{p k} \quad$ specific heat capacity of the $k$-th substance at constant pressure, $\mathrm{J} /(\mathrm{kg} \mathrm{K})$

$D_{j}, D_{k i} \quad$ diffusivity, $\mathrm{m}^{2} / \mathrm{s}$;

$E_{i} \quad$ activation energy for the $i$-th reaction, $\mathrm{J} / \mathrm{mol}$; $h=c_{p} T \quad$ overall enthalpy of the gas mixture, $\mathrm{J} / \mathrm{kg}$;

$J_{j \mathrm{~g}}^{\text {macro }}, J_{s \rightarrow \mathrm{g}}^{\text {macro }}$ macroscopic material fluxes caused by chemical reactions and phase conversions, $\mathrm{kg} /\left(\mathrm{s} \mathrm{m}^{3}\right)$;

$K_{e j}(T) \quad$ equilibrium constant;

$k \quad$ microscopic heat transfer coefficient, $\mathrm{J} /\left(\mathrm{m}^{2} \mathrm{~s} \mathrm{~K}\right)$;

$x_{*}$ reference length, $(\mathrm{m})$;

$\Psi=\frac{\lambda_{*}}{c_{*} \rho_{*}}$ temperature conductivity coefficient

$\mathrm{Pe}_{\mathrm{T}}=\frac{\mathrm{c}_{\mathrm{v}^{*}} \rho_{\star} \mathrm{x}_{*}^{2}}{\mathrm{t}_{*} \lambda_{*}}$ Thermal Peclet number;

$\mathrm{Ma}^{2}=\frac{\rho_{\star} \mathrm{u}_{*}^{2}}{\mathrm{p}_{*}}, \operatorname{Re}=\frac{\rho_{\star} \mathrm{X}_{*} \mathrm{u}_{*}}{\mu_{*}}=\frac{\rho_{\star} \mathrm{X}_{*}^{2}}{\mathrm{t}_{*} \mu_{*}} \quad, \quad$ Mach $\quad$ and

Reynolds numbers for gas component;

$G=\frac{g t_{*}^{2}}{x_{*}}$ Froude number;

$$
\begin{aligned}
\mathrm{N}_{\mathrm{E}} & =\frac{\varepsilon_{0} \mathrm{E}_{0}^{2}}{\rho_{*} \mathrm{u}_{*}^{2}}, \mathrm{~N}_{\varphi}=\frac{\mathrm{q}_{0} \mathrm{x}_{\star}^{2}}{\varphi_{0} \varepsilon_{0} \varepsilon}, \\
\mathrm{N}_{\mathrm{q}} & =\frac{\mathrm{j}_{0}}{\mathrm{q}_{0} \mathrm{u}_{\star}}, \quad \mathrm{N}_{\mathrm{J}}=\frac{\mathrm{j}_{0} \mathrm{E}_{0} \mathrm{t}_{\star}}{\rho_{\star} \mathrm{c}_{\mathrm{v}^{*}} \mathrm{~T}_{\star}}
\end{aligned}
$$

charge, and electrical current dimensionless parameters

$C_{p k}-$ specific heat capacity of substance $k$ at constant pressure, $\mathrm{J} /(\mathrm{kg} \mathrm{K})$;

$D_{j}, D_{k i}-$ diffusivity, $\mathrm{m}^{2} / \mathrm{s}$;

$E_{i}-$ activation energy for reaction $i, \mathrm{~J} / \mathrm{mol}$;

$h=c_{p} T-$ overall enthalpy of the gas mixture, $\mathrm{J} / \mathrm{kg}$;

$k$ - microscopic heat transfer coefficient, $\mathrm{J} /\left(\mathrm{m}^{2} \mathrm{~s} \mathrm{~K}\right)$;

$M_{j}-$ molar mass of specie $j, \mathrm{~kg} / \mathrm{mol}$;

$p_{*}$ reference pressure, $\mathrm{N} / \mathrm{m}^{2}$;

$Q_{i}^{S}$ - thermal effect for surface reaction $i, \mathrm{~kJ} / \mathrm{mol}$;

$R$ - universal gas constant, $\mathrm{J} /(\mathrm{mol} \mathrm{K})$;

$\left(\mathbf{S}_{\mathbf{V}}\right)_{i}=-u_{i} \kappa_{i}, \quad \kappa_{i}=\alpha_{i}|\mathbf{u}|+\beta_{i}, i=1,2,3 \quad-$ porous resistance, $\mathrm{kg} /\left(\mathrm{m}^{2} \mathrm{~s}^{2}\right)=\mathrm{N} / \mathrm{m}^{3}$;

$t$ - time, s;

$V(x, y, z)-$ mesovolume, $\mathrm{m}^{3}$

$V_{b}$ - volume of the condensed particle, $\mathrm{m}^{3}$;

$z_{k b}=\frac{l_{0}^{\prime} \rho_{0}^{\prime}}{b_{0}^{\prime} \rho_{p}^{\prime}}-$ particle growth, or evaporation, parameter,

dimensionless;

$\chi-$ porosity coefficient, dimensionless;

$\lambda$ is the thermal conductivity, $\mathrm{J} /(\mathrm{m} \mathrm{s} \mathrm{K})$;

$\mu_{0}^{\prime}-$ reference gas viscosity, $\mathrm{kg} /(\mathrm{m} \mathrm{s})=\mathrm{N} \mathrm{s} / \mathrm{m}^{2}$;

$\rho$ - gas density, $\mathrm{kg} / \mathrm{m}^{3}$;

SUBSCRIPTS

$S$-value at the particle surface;

* reference values.

\section{REFERENCES}

[1] Martirosyan K.S., Filimonov, I.A., Nersesyan, M.D., and D. Luss, Electric field formation during combustion of single metal particles, J. of Electrochemical Society, 150, 5, J9-16, 2003.

[2] Martirosyan K.S., Filimonov, I.A., and D. Luss, New Measuring Techniques of Electric Field Generated by Combustion Synthesis, Int. J. SHS, 11, 4, 325-333, 2002.

[3] Martirosyan K.S., Filimonov, I.A., and D. Luss, Self-heating by Joule dissipation during gas solid combustion reaction, Int. J. SHS, 12, 2, 91-98, 2003.

[4] Martirosyan, K. S., Filimonov, I. A., and Dan Luss "Electric Field Generation by Gas-Solid Combustion" AIChE J., 50, 1, 241-248, 2004. 
[5] Martirosyan K.S., M. Setoodeh and D. Luss, Electric field generated by the combustion of titanium in nitrogen, J. Appl. Phys., 98, 054901-6, 2005.

[6] Setoodeh M., K.S. Martirosyan, and D. Luss, Electrical pulse formation during high temperature reaction between $\mathrm{Ni}$ and Al. J. Appl. Phys., 99, 084901-7, 2006.

[7] Martirosyan K.S., Claycomb J.R., Miller J.H., and D. Luss Generation of the transient electrical and spontaneous magnetic fields by solid state combustion, J. Appl. Phys., 96, 8, 4632-4636, 2004.

[8] Martirosyan K.S., Claycomb J.R., G. Gogoshin R.A. Yarbrough Miller J.H., and D. Luss, Spontaneous magnetization generated by spin, pulsating and planar combustion synthesis, J. Appl. Phys., 93, 11, 9329-9335, 2003.

[9] Martirosyan K.S., D. Nawarathna, Claycomb J.R., Miller J.H. and D. Luss, Complex dielectric behavior during the formation of BaTiO3 by combustion synthesis, J. Phys. D: Appl. Phys., 39, 3689-3694, 2006.

[10] Filimonov I.A. and D. Luss, The High-Temperature Oxidation of a Metal Pellet: Nonisothermal Model, AIChE J., 51, 5, 1521-1575, 2005.

[11] Filimonov I.A. and D. Luss, Formation of Electrical Potential During the Oxidation of a Metal Particle, AIChE J., $50,2287,2004$

[12] Filimonov I.A., Kidin N.I., Formation of charged defects during the nitridation of a metal particle, Proceedings of the Combustion Institute, 31, 1991-1999, 2007.

[13] Filimonov I.A., Kidin N.I. On the Mechanism of Nitrogen Diffusion in Nitrides, Intern. J. of SHS, vol.14, no.3, pp. 157-171, 2005.

[14] Martirosyan K.S., and Luss D. Carbon Combustion Synthesis of Ferrites: Synthesis and Characterization. // Ind. Eng. Chem. Res. 2007. 46. 1492-1499.

[15] Martirosyan K.S. and D. Luss. Fabrication of metal oxides nanoparticles by highly exothermic reactions. // Chem. Eng. Technology. 2009. 32. 9. p.1376-1383.

[16] Martirosyan K.S., M. Iliev and D. Luss. Carbon combustion synthesis of nanostructured perovskites. // Int. J. SHS. 2007.16. 36-45.

[17] A.A. Markov, I.A. Filimonov, and K.S. Martirosyan. Simulation of front motion in a reacting condensed two phase mixture, J. Comput. Phys. Volume 231, Issue 20, 15 August 2012, Pages 6714-6724 (2012).

[18] A.A. Markov, I.A. Filimonov, and K.S. Martirosyan. Carbon Combustion Synthesis of Oxides: Effect of Mach, Peclet, and Reynolds Numbers on Gas Dynamics. Int. J. SHS. 2013, Vol. 22, No. 1, pp. 11-17.

[19] A.A. Markov, I. A. Filimonov, A. V. Poletaev, S. G. Vadchenko, and K. S. Martirosyan Generation of Charge Carriers during Combustion Synthesis of Sulfides. Int. J. SHS. 2013, Vol. 22, No. 2, pp. 69-76.

[20] Markov AA, Micro and macro scale technique for strongly coupled two-phase flows simulation// Computers and Fluids Volume/issue: 38/7 (2009) pp. 1435-1444.

[21] Markov A.A. Micro and Macro Scale Technique for Particle Growth Simulation// Computational Fluid Dynamics Review 2010. World Scientific 2010, Chapter 24, pp. 583-600.

[22] Markov, A.A. Multiscale Numerical Simulation of the Dispersed Reacting Flow, with Application to Chemical Vapor Deposition of Alumina. Proc. IV Intern. Conf. on Comput. Fluid Dynam. (ICCFD-4), Ghent 2006, SpringerVerlag, 2009, 753-758.

[23] Grigoriev Yu.M. and Filimonov I.A. Chemical Condensation in a Gas Flow. // (In Russian) J. Chem. Phys. 1994. 13. 10. 147-155.

[24] Nekrasov, B. V. course of General Chemistry. Goskhimizdat, 1960, 973 p. in Russian.

[25] Frank-Kamenetsky D.A. Diffusion and Heat Transfer in Chemical Kinetics in Russian M. Nauka . 1987491 p. 